\title{
e-VLBI Activities in Japan
}

\section{Sekido, ${ }^{a}$ N. Kawaguchi, ${ }^{b}$ Y. Koyama, ${ }^{c}$ M. Kimura, ${ }^{a}$ T. Kondo, ${ }^{c d}$ H. Takiguchi, ${ }^{a}$ T. Hobiger, ${ }^{c}$ K. Takefuji, ${ }^{a}$ H. Harai, ${ }^{c}$ T. Ikeda,${ }^{c}$ S. Shimojo, ${ }^{c}$ T. Oyama, ${ }^{b}$ T. Hara, ${ }^{b}$ Y. Kono, ${ }^{b}$ S. Kurihara, ${ }^{e}$ K. Kokado, ${ }^{e}$ D. Tanimoto,,${ }^{e}$ K. Nozawa, ${ }^{e}$ H. Takeuchi, ${ }^{f}$ H. Uose, ${ }^{g}$ K. Fujisawa, ${ }^{h}$ H. Takaba ${ }^{i}$}

${ }^{a}$ Kashima Space Research Center, National Institute of Information and Communications Technology, 893-1 Hirai Kashima Ibaraki, 314-8501, Japan, E-mail: sekido@nict.go.jp

${ }^{b}$ National Astronomical Observatory, 2-21-1 Osawa, Mitaka, Tokyo, 181-8588, Japan, kawagu.nori@nao.ac.jp

${ }^{c}$ Next Generation Network Research Center, National Institute of Information and Communications Technology, 4-2-1, Nukui-Kitamachi, Koganei, Tokyo 184-8795, Japan

${ }^{d}$ Ajou University, Paldai Hall 901, Worcheon-Dong, Yeongtong-Gu, Suwon 442-749, Korea

${ }^{e}$ Geographical Survey Institute, 1Kitasato, Tsukuba 305-0811, Japan

$f$ Japan Aerospace Exploration Agency/Institute of Space and Astronautical Science, 3-1-1 Yoshinodai, Sagamihara, Kanagawa 229-8510, Japan

$g$ Nippon Telegraph and Telephone Corporation, Musashino Research and Development Center, 3-9-11 Midori-cho, Musashino-shi, Tokyo, 180-8585, Japan

${ }^{h}$ Yamaguchi University, 1677-1 Yoshida, Yamaguchi, Yamaguchi, 753-8511, Japan

${ }^{i}$ Gufu University, 1-1 Yanagito, Gifu, Gifu, 501-1193, Japan

Recent Japanese e-VLBI activities are reported in this paper. Offline data transfer though the network has been regularly performed for geodetic VLBI experiments. Besides these offline transfers, real-time eVLBI experiments for ultra-rapid UT1 measurements have been performed among Onsala, Metsähovi, Tsukuba, and Kashima stations in a pilot project. In the field of astronomy, a real-time e-VLBI demonstration session organized by the ATNF in 2008 was the first occasion for Kashima to transmit VLBI data obtained by the K5 system to a foreign correlator in real-time. The global e-VLBI observation session organized by JIVE for the opening event of International Year of Astronomy in January 2009 was the first participation for a Japanese station in a global real-time e-VLBI session. Overview of the K5 DAS systems (K5/VSSP, and K5/VSI) used for the e-VLBI is described.

Optically linked e-VLBI observations with domestic VLBI stations have been conducted by the NAOJ. Also the NAOJ has developed a high speed VLBI data sender/reciver equipment named VOA-200 and a high speed hardware correlator (2Gbps $\times 6$ baselines). A test observation of 8 Gbps real-time VLBI was successfully achieved by using the VOA-200 and the hardware correlator in 2008.

Science and Technology of Long Baseline Real-Time Interferometry: The 8th International e-VLBI Workshop, EXPReSO9

June 22 - 262009

Madrid, Spain

\footnotetext{
* Speaker.
} 


\section{Introduction}

Development of a disk-based VLBI data acquisition system (DAS) named the K5 system was started in 2000 by the National Institute of Information and Communications Technology (NICT)[1]. The K5-system (K5/VSSP) had put its targets at (1) software correlation processing with a cluster of general purpose computers and (2) data transmission with IP protocol over the network. The K5/VSSP software correlation package developed by Kondo[2] supported not only processing of native K5/VSSP data files, but also Mark5A data files (Mk4 format) through data conversion. The K5/VSSP software package has been employed in JIVE (Joint Institute for VLBI in Europe) for fringe finding procedure to check the performance of radio telescopes before production EVN sessions. There is another kind of disk-based DAS system in the K5 family. The VSI (VLBI Standard Interface) compliant VLBI system named the K5/VSI was initially developed as a VLBI system oriented to high sampling rate observations[3]. A corresponding high speed software correlator, which has comparable performance with DiFX correlator[凹], for the K5/VSI has been developed by M. Kimura in 2003[5]. This software correlator is going to be used in the VERA project[6]. The K5 systems (K5/VSSP and K5/VSI) are currently used in not only domestic but also global e-VLBI observations both for geodesy and for astronomy. An overview of the K5 system is described in Section 2 . Recent international and domestic e-VLBI activities are presented in Section 3, and 4, respectively. Future prospects are given in Section 5 .

\section{Technology Developments (K5/VSSP and K5/VSI)}

The K5 is the name of a disk-based VLBI system including a DAS and a software correlator. There are two types of K5 systems, distinguished by the sampler and its data interface. One system is named the K5/VSSP, which was developed as multi-purpose sampler for scientific observations needing accurate time tags. VSSP is the acronym for Versatile Scientific Sampling Processor. One unit of the K5/VSSP32 sampler has 4 video signal inputs. Four units of the K5/VSSP are used as a set for a geodetic VLBI recording system with 16 video signal inputs. Available sampling modes of K5/VSSP are indicated in table 1. The software package for observation and data format conversion between K5/VSSP and Mark5 is freely available from the Internet ${ }^{1}$.

The other K5 system is the K5/VSI, which is characterized by a VSI-H interface. The VSI$\mathrm{H}$ is hardware specification of VLBI Standard Interface[7], which enables connection between different DASs such as the K5 and the Mark5. The K5/VSI was initially developed for astronomical

\begin{tabular}{|c|c|}
\hline Sampling rate $(\mathrm{MHz})$ & $\begin{array}{c}0.040,0.1,0.2,0.5,1, \\
2,4,8,16,32,64\end{array}$ \\
\hline Quantization bit & $1,2,4,8$ \\
\hline Number of Channels & 1 or 4 \\
\hline
\end{tabular}

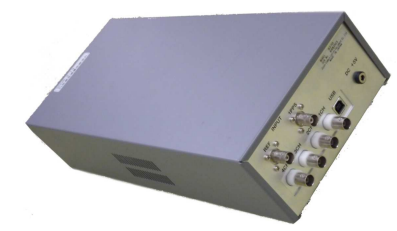

Table 1: Sampling mode of K5/VSSP32 (left) and one unit of K5/VSSP32 sampler(right). The sampler works with any combinations of these sampling parameters within maximum data rate of $256 \mathrm{Mbps}$.

\footnotetext{
${ }^{1}$ http://www2.nict.go.jp/w/w114/stsi/K5/VSSP/index-e.html
} 


\begin{tabular}{|c||c|c|c|c|}
\hline & ADS1000 & ADS2000 & ADS3000 & ADS3000plus \\
\hline \hline Sampling Rate & 1024Msps & 64Msps & 2048Msps & $4096 \mathrm{Msps}$ \\
\hline Quantization bit & 1 or 2 bit & 1 or 2 bit & $1 / 2 / 4 / 8$ bit & $1 / 2 / 4 / 8$ bit \\
\hline No. of Input & 1 & 16 & 1 & 4 \\
\hline No. of Channels & 1 & 16 & Programmable & Programmable \\
\hline Max Data Rate & 2048Mbps & 2048Mbps & 4096Mbps & $8192 \mathrm{Mbps}$ \\
\hline Interface & VSI-H(2 ports) & VSI-H(2 ports) & VSI-H(2 ports) & VSI-H (4 ports) \\
\hline
\end{tabular}

Table 2: Specifications of K5/VSI-Series Samplers

observations with the ADS1000 sampler. The multi-channel sampler ADS2000 and the digital baseband conversion sampler ADS3000/ADS3000plus are in the K5/VSI family. Table $⿴$ indicates the specification of K5/VSI samplers. The data stream from the VSI-H interface is captured into a computer by a PC-VSI card, which takes a VLBI data stream continuously through the PCI bus with a maximum data rate of up to 2048 Mbps.

\section{International e-VLBI}

\subsection{Ultra Rapid UT1 observation}

One of the benefits of using e-VLBI in the field of geodesy is to improve the latency in getting earth rotation parameters (EOP) derived from VLBI observation sessions. The understandings of the inner structure of the earth and the dynamics of the earth rotation are in progress, though accurate prediction of the earth rotation parameters is still difficult. In addition, VLBI is currently the only technique able to measure UT1-UTC, which represents the rotation angle of the earth with respect to the celestial reference frame fixed to a set of distant quasars, with long term stability. Improvement of the latency of VLBI analysis will contribute to predicting EOP with better accuracy.

As a pilot project to investigate the feasibility to use real-time VLBI data transfer in UT1 observations, Onsala Space Observatory, Metsähovi Radio Observatory, Tsukuba station of Geographical Survey Institute (GSI), and Kashima Space Research Center of NICT started a joint project for the quick derivation of UT1-UTC data with VLBI observations via real-time data transfer[8]. One of the issues was inhomogeneity of the DASs. Onsala and Metsähovi stations have been using Mark5A systems and Tsukuba and Kashima use K5/VSSP systems. The real-time data transfer from the Scandinavian stations to Japan was made by combination of the PC-EVN data capturing system [9] and a data transport protocol called Real-Time Tsunami[10]. The data format conversion from Mark5A(Mk4 format) to K5/VSSP and software correlation processing have been made by a K5/VSSP software package developed by Kondo. Sixty two sessions have been performed since March 2007 until July 2008. The session length for UT1 observation is usually one hour. Several observation modes: $512 \mathrm{Mbps}, 256 \mathrm{Mbps}$, and $128 \mathrm{Mbps}$ are tested in the sessions. A set of software to enable automated pipeline processing for data format conversion, correlation processing, MK3 database creation, and UT1-UTC estimation analysis has been developed in the project. Finally, the fastest record of UT1-UTC derivation was achieved on 21 February 2008, where UT1-UTC was obtained within 4 minutes after the end of the session. Based on the success of the project, we 

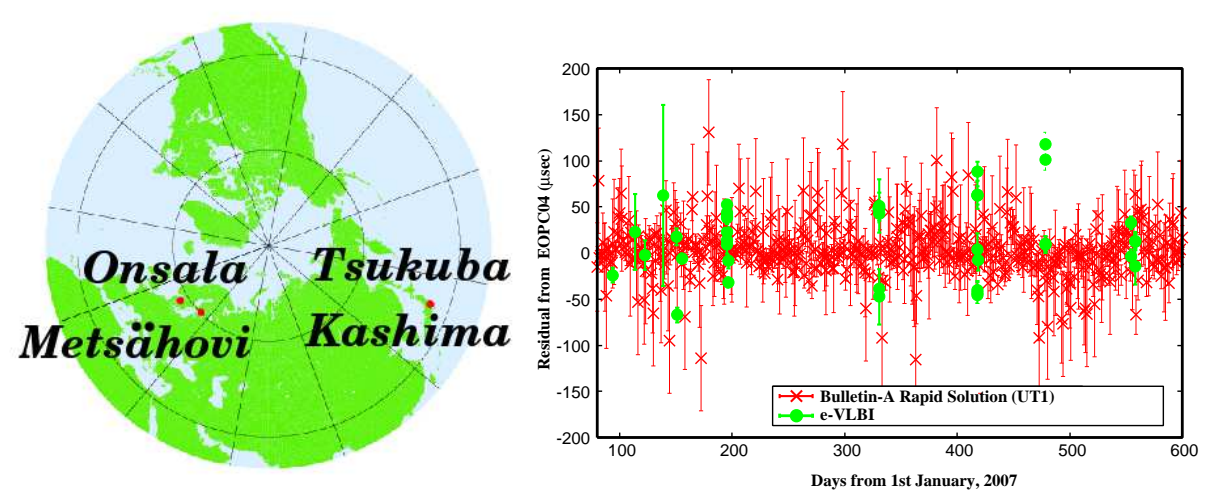

Figure 1: Stations of the Ultra Rapid UT1 sessions are in the left panel. UT1-UTC data derived by e-VLBI $(\bullet)$ and rapid solution of Bulletin-A $(\times)$ are plotted after UT1-UTC data of EOPc04 series are subtracted (right panel).

are preparing to use this technique for regular intensive UT1 observation sessions on the TsukubaWettzell baseline. Since Wettzell has a Mark5B system, which is VSI-H compliant, we are going to use a combination of [Mark5B] $+[\mathrm{K} 5 / \mathrm{VSI}]$ for data capture and transmission through the network. The data captured through the VSI-H connector will be transmitted over the network and format conversion to K5/VSSP will be done during the data transfer on the fly. Test observations and deployment in regular Intensive sessions should be done in this year.

As an extension of the pilot project, we are testing the feasibility of real-time data transfer and processing of 24h-long VLBI sessions for geodesy. The first trial of e-VLBI data transfer in a $24 \mathrm{~h}$ geodetic VLBI observation was made on 29 June 2009 by using the IVS session. The recording of observation data at a local site and real-time transmission of data through the network were done in parallel. This technique may drastically reduce the latency of 24-h geodetic VLBI observation results, which usually take about one week for collecting data and correlation processing at the correlation center.

\subsection{Participation to Global e-VLBI Observation}

Taking advantage of chances to participate in e-VLBI demonstrations, the compatibility of the K5/VSI system with JIVE and ATNF has been improved quickly in recent years. One of sessions was the e-VLBI demonstration in the 7th e-VLBI workshop held at Shanghai in June 2008. This VLBI session was organized by Australian Telescope National Facility (ATNF) with radio telescopes at Kashima, Shanghai, and Australia. The Kashima station participated by using a 'Mark5B data sender' developed by joint use of the ADS2000 sampler and the K5/VSI DAS. The VLBI data stream in Mark5B format was divided into small chunks of data and transmitted in UDP packets with a simple 64-bit sequential number attached to each packet as the header. The second chance was participation in the global e-VLBI demonstration for the International Year of Astronomy in 2009. We have improved the software from the 'Mark5B data sender' to that for a 'Mark5B emulator data server'. The data transmission function with UDP packets was unchanged, and the system was controlled via communication with a TCP/IP connection. The setting up of sampler mode and start/stop functions could then be controlled from a client (correlator). At every 

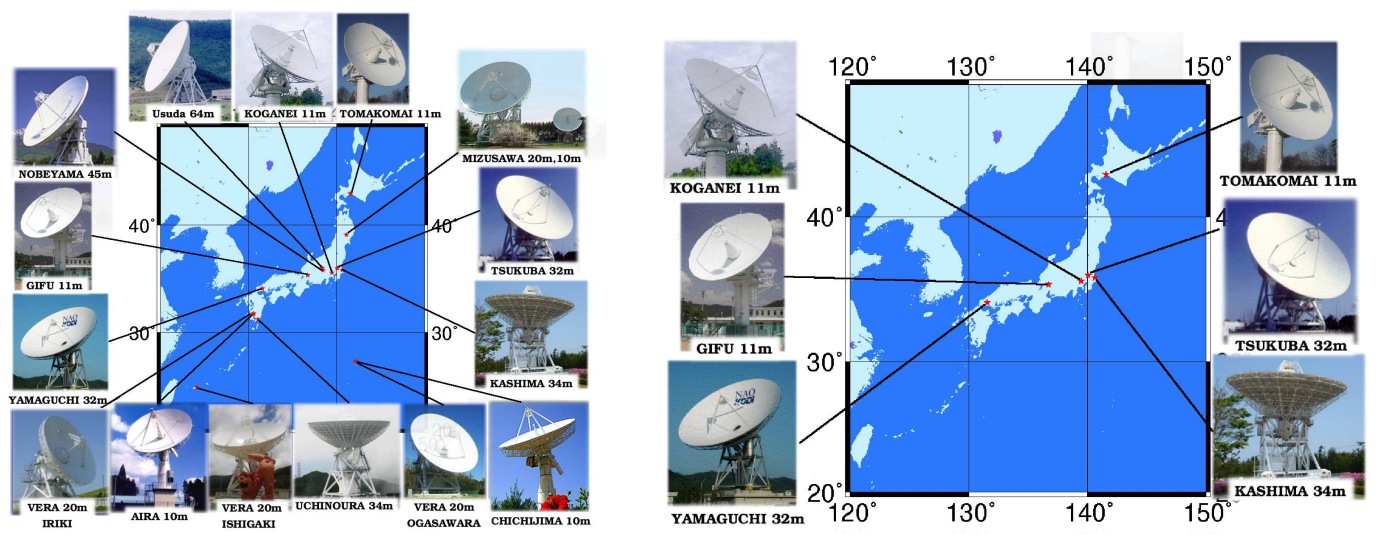

Figure 3: Japanese VLBI stations (left) and VLBI stations connected by high speed network (right).

occasion, compatibility issues are being fixed step by step. Improved compatibility of the DASs through this experience will be implemented in the $\mathrm{K} 5$ system, and will help future participation of Japanese stations in global e-VLBI observations.

\section{Domestic e-VLBI Activities}

Japanese VLBI activities are made up of contributions by NAOJ, NICT, GSI, JAXA/ISAS (Japan Aerospace Exploration Agency/Institute of Space and Astronautical Science), and Universities. Totally more than 15 VLBI stations are operated in Japan, although the number of telescopes connected by high speed networks is six at present (Fig.3). NAOJ has been conducting an optically linked high speed VLBI project. Astronomical VLBI observation sessions in $2 \mathrm{Gbps}$ (1Gsps/2bit/1ch) have been performed by the subset of the six stations. NAOJ has developed a real-time hardware correlator with 4-station 6-baselines processing capability (Fig.2). A VLBI network adapter named the VOA-200 has four VSI-H interfaces for input and output of data streams, and it works

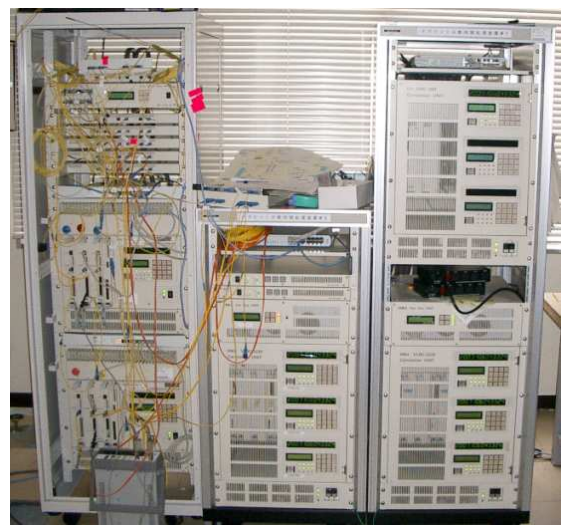

Figure 2: High speed hardware correlators with $2 \mathrm{Gbps} \times 6$ baselines capability(center-right) and VLBI network adapter VOA-200(left) developed by NAOJ. as both a sender and a receiver of VLBI data on the network. A series of test observations at 8096 Mbps (1024Msps/2bit/4ch) real-time VLBI with the VOA-200 and the hardware correlator were performed from May to June 2008 by using the 10 Gbps network of JGN2plus between Kashima-Koganei over a $100 \mathrm{~km}$ baseline. The test observations were successful and the VOA-200 demonstrated stable data transmission capability at $8096 \mathrm{Mbsp}$.

\section{Future Prospects}

The e-VLBI technology, using computers and networks has drastically reduced the difficulty for translating data format from one to the other. The K5/VSI system with the ADS2000 has become compatible with the Mark5B DAS by using the 'Mark5B Emulator' as described above. 
Moreover, the following format conversion schemes are in the scope of our development: (1)[ ADS2000 ]+[ PC-VSI ] $\Rightarrow$ [ Mark5B ], (2)[ Mark5B sampler ] + [ PC-VSI ] $\Rightarrow$ [ K5/VSSP ], (3) $[\mathrm{K} 5 / \mathrm{VSSP}] \times N \Rightarrow$ [ Mark5B ], (4) [K5/VSI] $\Leftrightarrow$ [VDIF], (5) [K5/VSSP] $\Leftrightarrow$ [VDIF]. Already case (1) is realized. Case (2) is in the plan to be used for real-time data transmission in regular UT1 observations between Wettzell and Tsukuba. Case (3) means transmitting K5/VSSP data to remote correlation site in Mark5B data format. Currently most of the Japanese VLBI stations have the K5/VSSP DAS. Thus this scheme will expand the opportunity for Japanese VLBI stations to participate in global VLBI observations.

The VLBI Data Interchange Format (VDIF) Specification [11] was ratified as a standard data VLBI format at the 8th international e-VLBI Workshop in June 2009. We are going to adapt K5/VSSP and K5/VSI systems to the VDIF format soon. When correlators become ready to accept VDIF data streams, the scheme (4) and (5) will be the targeted forms to be used in collaboration with world VLBI community.

\section{Acknowledgments}

The e-VLBI activities in Japan are supported by the high speed network environment provided by the Advanced Testbed Network for R\&D (JGN2plus), the Science Information Network (SINET3), and the test bed network GEMnet operated by NTT Co. Ltd.

\section{References}

[1] T. Kondo, et al.,Development of the new real-time VLBI technique using the Internet Protocol, IVS CRL-TDC News², 2000 (17) 22-24.

[2] T. Kondo, et al., Current Status of the K5 Software Correlator for Geodetic VLBI, IVS CRL-TDC News ${ }^{2}, 2003$ (23) 18-20.

[3] M. Kimura, J. Nakajima, H. Takeuchi, and T. Kondo, 2-Gbps PC Architecture and Gbps data processing in K5/PC-VSI, IVS CRL-TDC News ${ }^{2}, 2003$ (23) 12-13.

[4] A. Deller, S. J. Tingay, M. Bailes, C. West,A Software Correlator for Very Long Baseline Interferometry Using Multiprocessor Computing Environment, PASP 2007 (119) 318.

[5] M. Kimura, J. Nakajima, J. Ritakari, A. Mujumen., First Detection of 2-Gbps Wide-band Fringe between two PC-based VLBI Systems., IVS CRL-TDC News ${ }^{2}, 2003$ (22) 5.

[6] H. Kobayashi, et al., VERA System, Proc. of 7th EVN Symposium on VLBI Scientific Research \& Technology,2004, http://www.oan.es/evn2004/WebPage/HKobayashi.pdf

[7] A. Whitney, VLBI Standard Hardware Interface Specification - VSI-H, 2002, http://www.haystack.mit.edu/tech/vlbi/vsi/docs/2002_12_12_vsi-h_draft_rev_1.1.pdf

[8] M. Sekido, et al., Ultra-rapid UT1 measurement by e-VLBI, EP\&S 2008 (60) 865

[9] J. Ritakari, and A. Mujunen, Gbit/s VLBI and eVLBI with Off-The-Shelf Components, International VLBI Service for Geodesy and Astrometry 2004 General Meeting Proceedings (eds. Nancy

$R$. Vandenberg \& Karen D. Baver), NASA/CP-2004-212255, 182-185.

[10] M. R. Meiss, Tsunami: A High-Speed Rate-Controlled Protocol for File Transfer, 2004, http://steinbeck.ucs.indiana.edu/mmeiss/papers/tsunami.pdf.

[11] A. Whitney, M. Kettenis, C. Phillips, and M.Sekido, VLBI Data Interchange Format (VDIF) Specification, 2009

http://www2.nict.go.jp/w/w114/stsi/research/e-VLBI/VSI/VDIFspecificationRelease1.0ratified.pdf

\footnotetext{
${ }^{2}$ http://www2.nict.go.jp/w/w114/stsi/ivstdc/news-index.html
} 